\title{
SIFTing through satellite imagery with the Satellite Information Familiarization Tool
}

\author{
JORDAN J. GERTH \\ National Weather Service Office of Observations, National Oceanic and Atmospheric Administration, \\ Silver Spring, Maryland \\ RAYMOND K. GARCIA, DAVID J. HOESE, SCOTT S. LINDSTROM \\ Cooperative Institute for Meteorological Satellite Studies, Space Science and Engineering Center, \\ University of Wisconsin-Madison, Madison, Wisconsin \\ TIMOTHY J. SCHMIT \\ Advanced Satellite Products Branch, Center for Satellite Applications and Research, National Environmental \\ Satellite, Data, and Information Service, National Oceanic and Atmospheric Administration, Madison, Wisconsin
}

(Manuscript received 7 February 2020; review completed 8 June 2020)

\begin{abstract}
The Satellite Information Familiarization Tool (SIFT) is an open-source, multi-platform graphical user interface designed to easily display spectral and temporal sequences of geostationary satellite imagery. The Advanced Baseline Imager (ABI) and Advanced Himawari Imager (AHI) on the "new generation" of geostationary satellites collect imagery with a spatial resolution four times greater than previously available. Combined with the increased number of spectral bands and more frequent imaging, the new series imagers collect approximately 60 times more data. Given the resulting large file sizes, the development of SIFT is a multiyear effort to make those satellite imagery data files accessible to the broad community of students, scientists, and operational meteorologists. To achieve the objective of releasing software that provides an intuitive user experience to complement optimum performance on consumer-grade computers, SIFT was built to leverage modern graphics processing units (GPUs) through existing open-source Python packages, and runs on the three major operating systems: Windows, Mac, and Linux. The United States National Weather Service funded the development of SIFT to help enhance the satellite meteorology acumen of their operational meteorologists. SIFT has basic image visualization capabilities and enables the fluid animation and interrogation of satellite images, creation of Red-Green-Blue (RGB) composites and algebraic combinations of multiple spectral bands, and comparison of imagery with numerical weather prediction output. Open for community development, SIFT users and features continue to grow. SIFT is freely available with short tutorials and a user guide online. The mandate for the software, its development, realized applications, and envisioned role in science and training are explained.
\end{abstract}

\section{Introduction}

The Satellite Information Familiarization Tool (SIFT) is freely available open-source software that is based on a graphical user interface (GUI) for displaying, animating, and interrogating images from "newgeneration" geostationary weather satellites, requiring little to no experience with software programming languages to use. SIFT development began at the
University of Wisconsin-Madison Space Science and Engineering Center (SSEC) in 2015. The acronym references a famous "sifting and winnowing" plaque on the University's Bascom Hall that commemorates academic freedom. Satellite meteorologists sift and winnow through countless weather satellite images to uncover the details of the atmosphere and its processes.

SIFT was borne from a rapid prototyping project to build proof-of-concept software for handling 
imagery from the Japan Meteorological Agency (JMA) Advanced Himawari Imager (AHI), the first advanced weather imager in geostationary orbit, with 16 spectral bands (Bessho et al. 2016, Okuyama et al. 2018). The United States National Weather Service (NWS) sought a workshop on operational applications of AHI imagery for their meteorologists in Guam, and due to the later launch of the first satellite in the Geostationary Operational Environmental Satellite $R$-Series (GOES-R), a training software application and associated materials had not yet been developed. This presented the unique opportunity to design and develop SIFT based on early operational user training needs for the western Pacific Ocean, a data-sparse portion of the world particularly reliant on geostationary weather satellites for weather monitoring.

Eventually, SIFT was integrated into training sessions for more than 100 NWS science officers as part of a recurring short satellite meteorology residence course at the NWS Training Center, offered seven times in late 2016 and 2017. This course offering approximately coincided with the availability of data from the first GOES-R satellite, GOES-16. Since that time, SIFT has evolved to serve a broadening set of users within the United States and beyond. It has been used with American university students to explain the potential applications for geostationary satellite imagery in atmospheric research, and for international training activities in South America, where United States satellites are used routinely in weather monitoring.

Although initially developed with certain training objectives in mind, the capabilities of SIFT make it a versatile tool for the operational meteorologist or atmospheric scientist to use regularly. There were three principles that guided the development of SIFT: the software must be free and open source, based on a user interface that is simple and intuitive to use, and available for multiple major operating systems. SIFT is implemented in Python and has been packaged for Windows, Mac, and RedHat/CentOS Linux. It is distributed under the GNU General Public License v3 (GPLv3) with installers. The development of SIFT has also focused on delivering the best possible user experience for their hardware; this required shifting major manipulations of large image data arrays to the graphics processing unit (GPU).

There are other meteorological satellite visualization packages available. However, none of the existing packages were intended for an expanded set of users that SIFT serves. For example, the Advanced
Weather Interactive Processing System (AWIPS) that the NWS uses is enterprise software that is difficult for a single user to install and does not allow for opening archived data without reprocessing. Some international meteorological agencies also develop or use specialty visualization software that is difficult to obtain or obtain data for. And while SIFT shares some common features with the free Man computer Interactive Data Access System-V (McIDAS-V), SIFT is narrowly tailored to handle particular types of weather satellite data and incorporates the data analysis and interrogation features that users of operational weather satellite data are most likely to need.

SIFT capabilities minimize the amount of time necessary, and number of user actions required, to load a set of satellite image data files. SIFT users can also easily export images or animations of suitable quality for publications and presentations. Like other software that relies on a GPU, SIFT performs best on high-performance desktop computers, though it runs adequately on consumer-grade personal computers. At a minimum, SIFT requires a 64-bit operating system with at least $256 \mathrm{~GB}$ of free disk space, $8 \mathrm{~GB}$ of RAM, and 2 GB of video RAM with OpenGL 3 support. A solid-state disk (SSD) is preferred to store the software and raw data.

This article will describe the features of SIFT; the development strategy, including the architecture and performance considerations; and some applications based on training events that have incorporated SIFT into learning exercises. The capabilities of SIFT will continue to grow in the future as more geostationary satellites are launched and users desire additional features for interrogating imagery and products from those satellites. The intent of this article is to introduce SIFT such that it entices readers from across the meteorological community and around the world to download SIFT for the purpose of incorporating it into their research, operations, and educational activities, regardless of previous experience with meteorological satellite data.

\section{Capabilities and features}

The SIFT GUI has three major components: a map display, a layer list, and a layer details panel. An example of the GUI can be found in Fig. 1. There is a supplemental video that provides a brief introduction to SIFT and its capabilities for loading and interrogating GOES-16 Advanced Baseline Imager (ABI) imagery. 


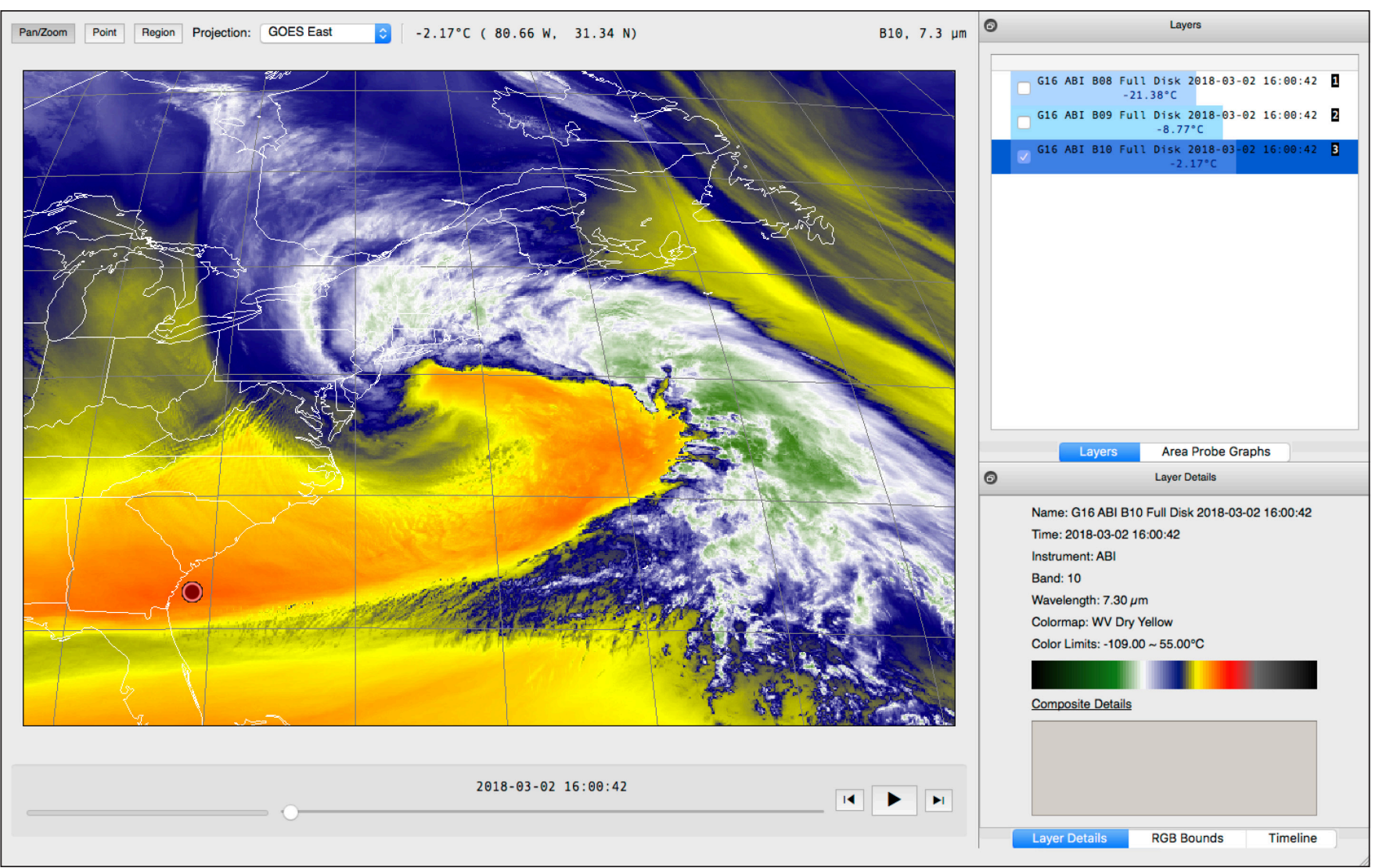

Figure 1. This is a screen capture of the SIFT graphical user interface (GUI). The GUI has three main components: a map display, a layers list, and a layer details panel. The menu system is not shown. Users can change the projection and areal extent of the map display. Here, three GOES-16 Advanced Baseline Imager (ABI) water bands are loaded; one is visible on the map display. The red dot is the probe. Click image for an external version; this applies to all figures and hereafter. The narrated video accompanying this figure shows the SIFT graphical user interface and demonstrates several SIFT features in loading and interrogating GOES-16 ABI imagery during a dust event.

SIFT has a menu system for loading data and modifying characteristics of the displayed data and map background. Experienced users can make use of action keys associated with the menu items to perform simple tasks, such as altering the color and visibility of political boundaries and grid lines. For complex or long operations, such as loading multiple data files, SIFT contains a background task status indicator. More on the map display and coordinate transformation is explained in the subsequent section on the architecture.

\section{a. Animating imagery}

The major benefit of geostationary satellite imagery compared to polar-orbiting satellite imagery is the ability to create animations from images that are consecutive in time. Therefore, one of the most important SIFT features is the ability to seamlessly loop across multiple times. SIFT is also able to loop across multiple spectral bands for a single time. The animation capabilities allow users to identify similarities in the appearance of certain scene features across spectrally adjacent bands and times. There is an animation control that enables users to step through individual frames or "autoplay" them with an adjustable speed control. Users can continue to pan and zoom while animating a sequence of images.

With the advanced geostationary imagers, the most difficult aspect for new users to learn is the unique properties of the spectral bands and their applications. The ABI, similar to the AHI, has sixteen spectral bands, including ten in the infrared. Full disk imagery from these advanced imagers are captured every 5 to 15 minutes, a regional sector every 2.5 to 5 minutes, and mesoscale images (two sectors per ABI) every minute. The spatial resolution is no greater than $2 \mathrm{~km}$ (infrared 
bands) at the satellite subpoint (Schmit et al. 2005, 2017; Kalluri et al. 2018). Given the range of interesting meteorological phenomena that the geostationary imagers capture, SIFT provides users with an interface to load and animate data that enhances learning of how phenomena of interest appear in different spectral bands and evolve with time.

\section{b. Enhancing and compositing imagery}

An important aspect of visualizing satellite data is constructing a color enhancement that reliably depicts and distinguishes features of interest of varying properties, such as cloud, snow, or vegetation, using the observational data (Gerth 2019). For an easy user experience, modifying the visible range and color enhancement for a certain spectral band at one loaded time will also update the same display characteristics for other loaded times. Furthermore, SIFT is packaged with several color enhancements from AWIPS that are used in NWS forecast offices. Users can also create their own color enhancements through a gradient editor interface, which can also import and export enhancements. Also, users have the option to include a corresponding labeled color enhancement bar on images saved using SIFT.

SIFT can combine multiple bands for the same time through a user-defined arithmetic composite, such as a band difference, or a Red-Green-Blue (RGB) composite (d'Entremont and Thomason 1987), which combines spectral bands and sometimes multi-band composites for a single time into a false color image. The RGB composite features allow users to customize the three components, with the capability for users to constrain the ranges of each contributing band and set a gamma adjustment (the power/curvature to use in translating value to color intensity) to enhance one end of the component range more than another. SIFT can easily create an animation of RGB composites for multiple times based on the initial formulation and the availability of the necessary input layers.

\section{c. Interrogating or probing imagery}

SIFT operates on a layer stack display system, similar in some aspects to AWIPS and Adobe Photoshop. Each unique image or field that the user loads, or composite that the user creates, is a layer in SIFT. The layer list panel enables users to reorder or remove layers, toggle the visibility of a layer on the map display, and modify layer properties such as the visible range and color enhancement. The layer details panel contains the metadata for the selected layer, including band information, the applied color bar, and range limits.

Users have the basic capabilities to interrogate the layer data using a three-button wheel mouse or equivalent. SIFT has three major capabilities that enable user interaction with specific portions of a layer: a pan and zoom feature for clearly viewing a portion of the image on the map display; a point probe that can be dropped at any pixel to reveal the spatial coordinates (e.g., latitude and longitude) and corresponding data value; and an area, or region, selector to build a polygon for which comparison queries can be run and displayed in graphs.

The comparison queries of the data within the userdefined polygon are rendered in the form of a graph, known as an area probe graph. Users can specify the desired type of area probe graph from two options: a histogram showing the number of values in each interval, or a density (binned scatter) plot that collocates the corresponding values from two layers (discussed with an example in section 4). The most practical use of this feature is to compare the reflectance or brightness temperature distribution for different scenes and multiple spectral bands. The user can create multiple graphs from the same area selection polygon for further band-to-band comparisons, or different polygons on the same layer(s) for feature comparisons. Exporting graph figures is supported; axis information and labeled color bars are customizable.

SIFT can handle a point probe and polygon simultaneously on the map display. When the probe is placed in the map display, the layer list shows the value at the probe point for all listed layers regardless of their visibility. The point probe value also appears on area probe graphs, distinguished from the other data.

\section{Development}

SIFT is built on Python and relies on several renowned open-source Python science packages to load and render satellite data. Python was selected for SIFT because it is a cross-platform language of growing use in the atmospheric sciences. This section describes the technical architecture, including the Python packages and libraries leveraged in SIFT and performance aspects of handling and visualizing a series of large geospatial data files. 


\section{a. Architecture and performance}

SIFT's overriding purpose is to permit fluid, intuitive interaction with dense geospatial satellite imagery, including time animations. To achieve this, development initially focused on demonstrating that large image datasets, those larger than can be held in memory, could be effectively transported from disk to GPU texture buffers. Projection calculations, colorvalue mapping, and composition are offloaded to shader code aboard the GPU, using full dynamic-range input data. By performing these operations in the GPU, modifications are reflected immediately on-screen. Doing the same with a CPU implementation would require multiple seconds or minutes to reprocess the image data with even small changes.

There are two main components of the SIFT architecture, as shown in Fig. 2: an on-disk cache and a GPU rendering container. SIFT uses a database in tandem with a disk cache of recent data loaded from files, independent of session, to ease the recovery of frequently referenced images. When a new file is loaded, it is first cached on the disk prior to GPU rendering. The most resource-intensive images for SIFT to load are the $0.5-\mathrm{km}$ nadir-resolution full disk visible bands, each one consuming up to 2 GB in size when uncompressed on disk; each hour of data, consisting of just four to six images, can approach or exceed 10 GB. The 2-km infrared band images, consisting of one-sixteenth of the

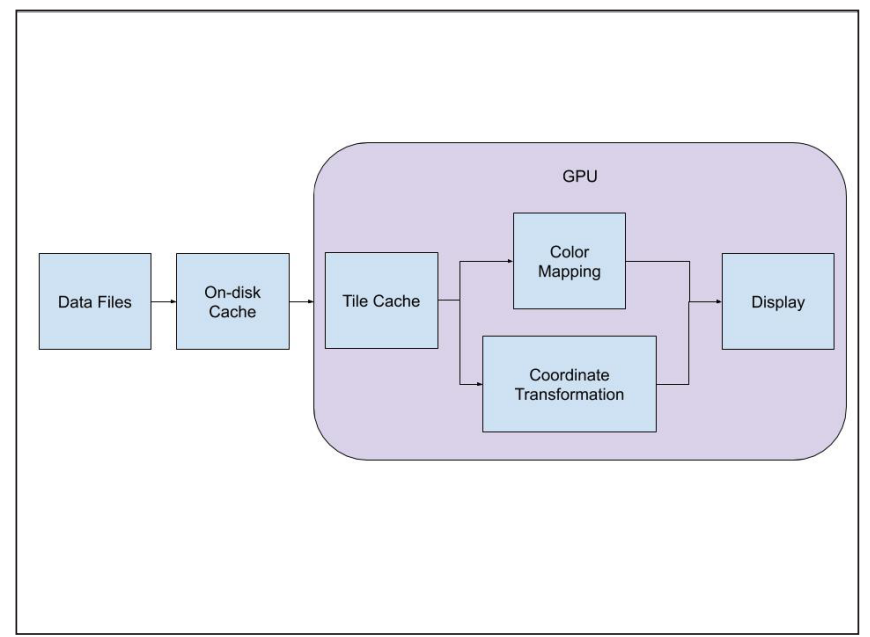

Figure 2. This diagram shows the handling of data through SIFT to the display. Data is first cached on disk and then rendered on the graphics processing unit (GPU). The rendering process includes caching, coloring, and transforming the data for the user-desired display. number of pixels, are a fraction of that, up to $125 \mathrm{MB}$ uncompressed on the disk. These are relatively large images to process and keep in memory for SIFT to provide an interactive user interrogation experience.

To render a sequence of large images to the SIFT map displays, data files are first read, written to an ondisk cache, then accessed for visualization from the cache as memory-mapped files. Memory mapping is an operating system feature that allows portions of large data arrays to transparently move in and out of memory. Subsets are then copied to the GPU memory as textures for tile mapping, coordinate transformation, and rendering. For this reason, the SIFT on-disk "workspace" is best implemented using a SSD commensurate with the data volume. This architecture allows the operating system to rapidly bring the most-needed imagery data into RAM (CPU memory) prior to loading on the video RAM (GPU memory) as a background operation. It takes advantage of the large disk storage that is slow compared to the CPU memory, which in turn is large but slow compared to GPU memory. The user can operate on the data available while SIFT updates sections and slices. SIFT selects the highest-resolution data that are most optimal for the user's changing map display view.

To accomplish that, the GPU texture buffers are dynamically populated with data. Textures are updated to a resolution proportional to that of the screen to keep a stable CPU and GPU memory footprint, as well as GPU calculation load while allowing the user to fluidly pan, zoom, and animate the sequence. The ABI 16-channel imagery contains 12 to 14 bits of dynamic range for up to 22 000-pixel square image arrays covering the full disk of the Earth observable from the satellite. Resource-constrained users can also retrieve and load smaller sectors, such as the CONtiguous United States (CONUS) and mesoscale sectors that are part of one of the ABI's scan modes.

\section{b. Data}

SIFT can read network Common Data Form (netCDF) "L1b" radiance (or cloud and moisture imagery) files that the GOES-R ground system produces. $G O E S-R$ files of this format are hosted on the National Oceanic and Atmospheric Administration (NOAA) Comprehensive Large Array-data Stewardship System (CLASS) at www.class.noaa.gov/. The "L1b" files from the GOES-R ABI are also found on major cloud services. These data from the United States government weather satellites are free and accessible. 
Table 1. This table lists, alphabetically, the major Python science package and library dependencies of SIFT, along with their purpose and specific usage within SIFT.

\begin{tabular}{|c|c|c|c|}
\hline Name & Purpose & SIFT Usage & Reference \\
\hline Imageio & $\begin{array}{l}\text { Easy Pythonic image and } \\
\text { video generation }\end{array}$ & $\begin{array}{c}\text { Export animations and images using } \\
\text { FFmpeg and Pillow }\end{array}$ & Klein et al. (2018) \\
\hline Matplotlib & Interactive scientific plotting & Probe graphics & Hunter (2007) \\
\hline Numba & $\begin{array}{c}\text { Numerical expression } \\
\text { compiler }\end{array}$ & $\begin{array}{l}\text { Efficient probe area extraction and } \\
\text { data transforms }\end{array}$ & Lam et al. (2015) \\
\hline NumPy & $\begin{array}{l}\text { Efficient array and matrix } \\
\text { math }\end{array}$ & $\begin{array}{c}\text { Data array container and efficient } \\
\text { calculations; fast memory-mapped } \\
\text { data caching and access }\end{array}$ & Oliphant (2006) \\
\hline PyProj & $\begin{array}{c}\text { Geographical Information } \\
\text { System (GIS) map projection } \\
\text { math }\end{array}$ & Coordinate systems transforms & Whitaker \\
\hline SatPy & $\begin{array}{l}\text { Meteorological satellite data } \\
\text { processing Python library }\end{array}$ & $\begin{array}{l}\text { Read Gridded Binary (GRIB) and } \\
\text { other satellite data formats }\end{array}$ & $\begin{array}{l}\text { Raspaud et al. } \\
\quad(2018 \mathrm{a}, \mathrm{b})\end{array}$ \\
\hline VisPy & $\begin{array}{l}\text { High-level OpenGL Python } \\
\text { library }\end{array}$ & Map and data visualization & $\begin{array}{c}\text { Campagnola et al. } \\
\text { (2018) }\end{array}$ \\
\hline
\end{tabular}

The data import framework for SIFT is increasingly using the SatPy library (Raspaud et al. 2018a,b), which supports a broad range of meteorological satellite data as well as a subset of numerical weather prediction (NWP) fields, for data handling. Incorporating SatPy has unlocked user access to a wider range of weather satellite data. For example, lightning data from the GOES-R Geostationary Lightning Mapper (GLM) is readable with SIFT using SatPy. SIFT can also load Himawari Standard Data (HSD) and HimawariCast High Rate Information Transmission (HRIT) files, as well as European Meteosat Spinning Enhanced Visible and InfraRed Imager (SEVIRI) and Republic of (South) Korea Advanced Meteorological Imager (AMI) "L1b" imagery. Although displaying gridded NWP output is not a primary capability of SIFT, SIFT has a basic interface for loading a Gridded Binary (GRIB2) file and contouring a specific field to match with a satellite image layer corresponding in time. GRIB2 field units are attributed to a layer using tables found in the SIFT dependencies.

\section{c. Python packages}

Including SatPy, SIFT has seven major software package dependencies that are listed in Table 1, along with their role in SIFT's capabilities. VisPy provides the core application capability, which drives the map display, an Open $G L$ canvas with a graph of visual elements to show on the screen. Using the GPU, this canvas supports panning and zooming across the loaded image layer, with the image resolution dynamically rendered based on the zoom level. The map display incorporates predefined geostationary, Lambert Conformal, Mercator, and Polar Stereographic projections. SIFT remaps data from the native projection in the data files to any of those aforementioned projections preconfigured in the software. The PyProj module provides a Pythoncallable interface to the popular PROJ map projection $\mathrm{C}$ library.

In tandem with NumPy providing data slices that are fed into the GPU texture storage, polygons representing the PROJ-compatible coordinate reference system (CRS) information are rendered using a variety of pre-existing and custom graphics language shader code running on the GPU. This allows, for example, color-value map calculations in real-time using the full dynamic range of science data present in the texture atlas.

Satellite data are loaded into SIFT maintaining its native units, with a preference for Celsius where Kelvin may otherwise be used. For visible and near-infrared spectral band data, the default unit is a reflectance ratio. Area and point probes require inverse projection and data sampling using PyProj, NumPy, and Numba to perform data masking and extraction. The probe data are then fed to Matplotlib, a MATLAB-inspired generalized plotting toolbox to produce histogram and 


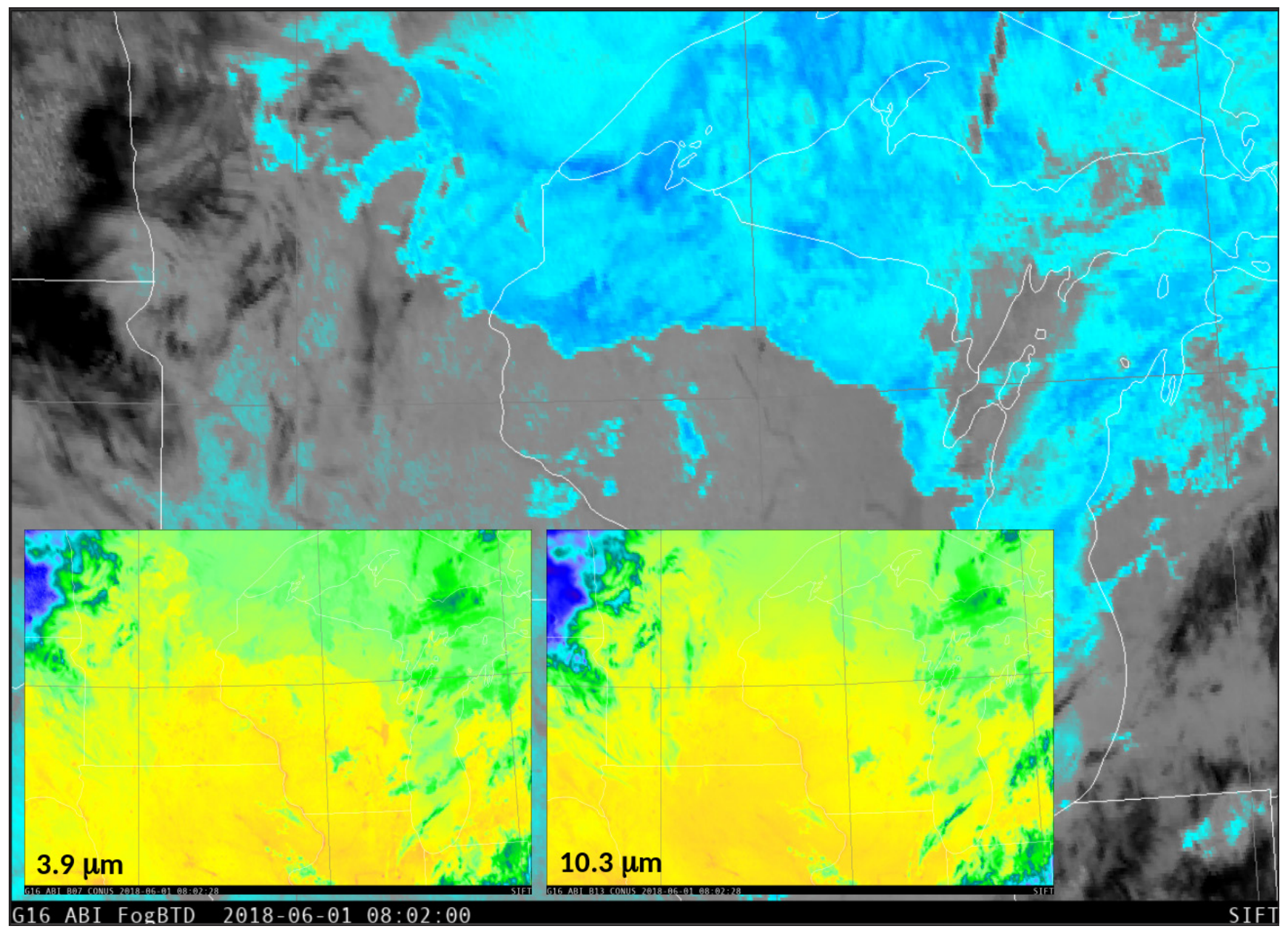

Figure 3. This GOES-16 Advanced Baseline Imager (ABI) "Night Fog" brightness temperature difference field $(10.3 \mu \mathrm{m}-3.9 \mu \mathrm{m})$ at 0802 UTC on 1 June 2018 depicts low clouds comprised of water droplets (enhanced in blue over northern Wisconsin, the upper peninsula of Michigan, and surrounding areas) and also higher cirriform clouds over the eastern Dakotas (black in the enhancement). Inset images show the individual bands (with the same rainbow color enhancement applied) from which the difference field is created.

scatter plots, which can be saved. The Imageio library is used to generate and save image files of individual animation frames or full animation videos from the map display.

The GUI elements are provided by the crossplatform Qt5 library, which allows the application to operate on recent Windows, Mac, and Linux systems with minimal code specific to the operating system. Qt5 is bridged to Python using PyQt5. There are many other open source packages that SIFT leverages for the user interface, software management and packaging, and basic capabilities (Space Science and Engineering Center 2019).

\section{Applications}

Meteorologists can use SIFT's capabilities to easily display single bands, channel differences, and RGB composites to diagnose the state of the atmosphere in case studies. With SIFT, users can explore and compare elements of the data. SIFT enables users to easily create spectral band combinations based on an arithmetic formula without substantial coding or configuration. A band difference is among the simplest of spectral band combinations. Specific instructions for a new user to create and interrogate a band difference using SIFT can be found in Appendix A. 
The common and aptly named "Night Fog" brightness temperature difference field comprises 10.3$\mu \mathrm{m}$ and $3.9-\mu \mathrm{m}$ brightness temperature image layers, where the latter is subtracted from the former. SIFT can compute and render this difference field, like each usergenerated arithmetic field, as a new layer after the input layers (in this case, the individual bands) are loaded and rendered. The background SIFT screen capture in Fig. 3 shows the "Night Fog" brightness temperature difference field over Wisconsin and surrounding states at 0802 UTC on 1 June 2018. For this nighttime case and particular color enhancement, an extensive stratus cloud field appears in hues of blue, where the 10.3- $\mu \mathrm{m}$ brightness temperatures are greater than the collocated 3.9- $\mu \mathrm{m}$ brightness temperatures (i.e., the difference is positive), extending from northwestern Wisconsin to the east and south into central Lake Michigan. The cloud in eastern Wisconsin is the leading edge of a southward-moving "pneumonia"-type front, a regional, southward-driving phenomena that sharply separates warm and humid air with seasonably cooler, drier, and more dense air from the Great Lakes.

Insets in Fig. 3 show 3.9- $\mu \mathrm{m}$ (left) and $10.3-\mu \mathrm{m}$ (right) brightness temperatures with the same rainbow color enhancement and value correspondence. The

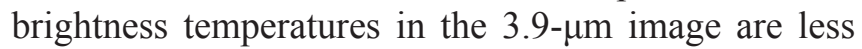
in regions where stratus clouds are present due to their water droplet composition, underscoring the concept that cloud droplet emissivity is a function of wavelength

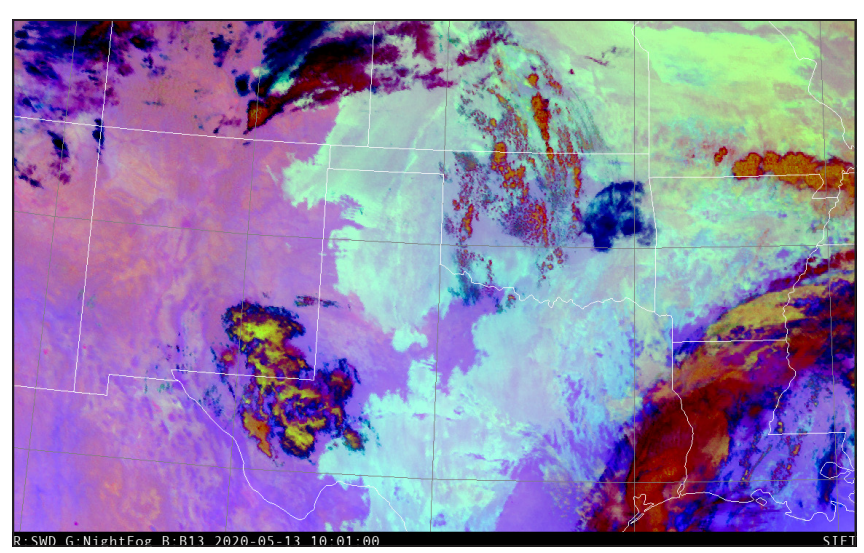

Figure 4. This GOES-16 Advanced Baseline Imager (ABI) nighttime microphysics Red-Green-Blue (RGB) composite, based on Lensky and Rosenfeld (2008) and exported from SIFT, at 1001 UTC on 13 May 2020 depicts an expansive area of low cloud (tint of aqua) over the Southern Plains of the United States. Review more imagery from this event online: fusedfog.ssec. wisc.edu/?p=4299.
(Ellrod 1995). In addition to rendering a plan view image, SIFT offers users a point probe for interrogating individual pixels to quantify the brightness temperature difference, in this case, between two qualitatively similar infrared window bands for a stratus cloud because of wavelength-dependent emissivity differences.

The "Night Fog" field is the green component of the nighttime microphysics RGB composite that is also commonly used for fog and low stratus detection (Lensky and Rosenfeld 2008). SIFT can produce common RGB composites such as the nighttime microphysics RGB composite shown in Fig. 4. User familiarity with band composites portrayed as a RGB composite can speed identification of clouds without requiring the interrogation of multiple bands. SIFT users can easily change input band components for the RGB, or adjust the value range over which a contrast in component contribution is applied, to create custom RGB composites. Low stratus in the nighttime microphysics RGB appears a tint of aqua, such as over southwestern Kansas and the Oklahoma and Texas panhandles; high cirrus is purple, such as over eastern Colorado and western Louisiana; and developing cumulonimbus are orange or red, such as over central Kansas.

SIFT also allows a user to outline a region with a polygon to compare spectral bands. An example of a pixel-wise comparison of 3.9- $\mu \mathrm{m}$ (Band 7) and 10.3$\mu \mathrm{m}$ (Band 13) brightness temperatures is shown in the density plot in Fig. 5. The comparison includes pixels in a region that includes stratus cloud and clear skies at the same time as in Fig. 3. Two relationships are evident: in clear skies, the brightness temperatures are approximately equal and are clustered along the line $x=$ $y$; in low clouds, $3.9-\mu \mathrm{m}$ brightness temperatures are less than those at $10.3 \mu \mathrm{m}$ because of emissivity differences from cloud droplets at those two wavelengths.

Many different investigations into the atmosphere and weather phenomena are possible with SIFT. Research into new applications for advanced geostationary weather imager data continues (Schmit et al. 2018). SIFT can be used to confirm and expand the use of those applications, as well as identify new ones. Beyond the procedure included in the appendix, SIFT laboratory exercises are available on the SIFT web sift.ssec.wisc.edu/. These exercises allow users to explore a range of remote sensing applications with the new geostationary imagers, such as the utility of the split window difference $(12.3 \mu \mathrm{m}-10.3 \mu \mathrm{m})$ field for identifying conditional convective instability. 


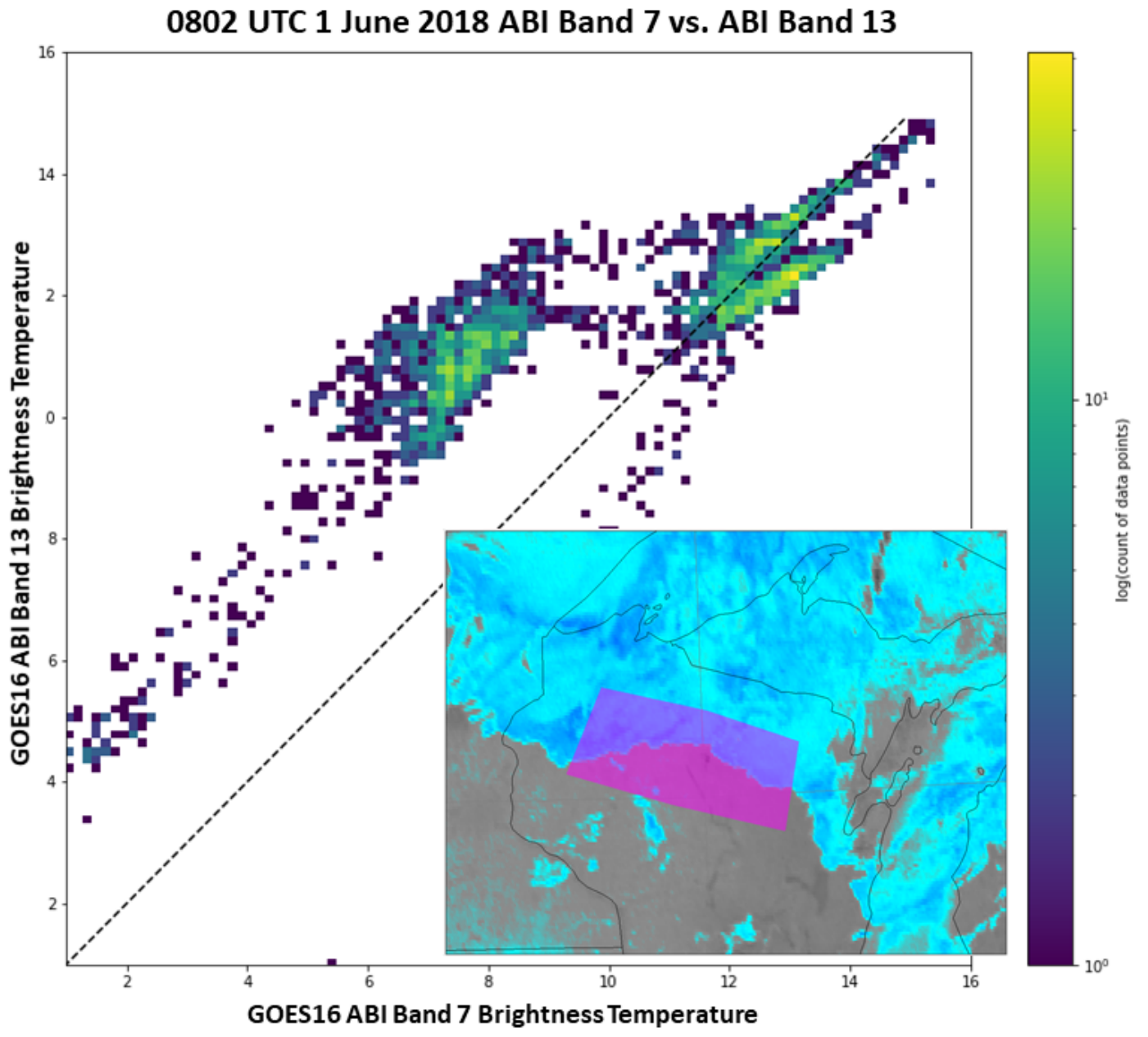

Figure 5. This density plot compares the GOES-16 Advanced Baseline Imager (ABI) 3.9- $\mu$ m shortwave infrared window band 7 ( $x$-axis) brightness temperatures $\left({ }^{\circ} \mathrm{C}\right)$ with $\mathrm{ABI} 10.3-\mu \mathrm{m}$ clean infrared window band 13 ( $y$-axis) brightness temperatures $\left({ }^{\circ} \mathrm{C}\right)$ for collocated points within the polygon outlined in the inset "Night Fog" brightness temperature difference field from 0802 UTC on 1 June 2018 (also shown in Fig. 3). The dashed line corresponds to where brightness temperatures of the contributing spectral bands are equal (i.e., $x=y$ ).

\section{Future directions}

There is a small but growing community of SIFT users of GOES-R and Himawari imager data. There is also a desire to enhance SIFT to support all geostationary weather satellite missions with accessible visualization for data. Recent additions to SIFT include support for the Republic of (South) Korea Advanced Meteorological Imager (AMI), similar to the AHI, and the ability to load and render data from the GOES-R Series Geostationary Lightning Mapper (GLM).

There are two major benefits for increasing the number of satellites and data formats that SIFT uses. First, support for more satellites increases the possibilities for inter-calibration and validation. For example, there is an overlap in coverage between adjacent geostationary satellites, such as Himawari and the GOES-R satellites that are in orbit. Second, many SIFT-based lab activities developed for users of one advanced geostationary imaging satellite can easily be adapted to support another mission.

SIFT continues to be used in training events geared toward meteorologists and students. Given the maturity, stability, and accessibility of SIFT, it is suitable for classroom labs at universities to complement coursework. Installers, short tutorials, and a user guide

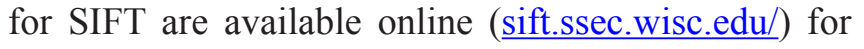
students and instructors. 
The feature request list for SIFT remains long. Software developers can contribute code to the SIFT base through GitHub (github.com/ssec/sift/). A wider effort toward refinement and further modularization of the software is ongoing with a goal of making the code base inviting to open-source contributors, as well as improving the feasibility of implementing new capabilities under the designed architecture.

\section{Summary}

The "new generation" of advanced geostationary satellite imagers has provided a need for consumergrade software to improve the accessibility of the data for users in the meteorological community and beyond. The development of SIFT fills this void. SIFT is built on Python for cross-platform support, uses the GPU to render imagery at full spatial resolution (down to $0.5 \mathrm{~km}$ ) without sacrificing performance, and is freely distributed under the GNU GPLv3 license. SIFT has been used to demonstrate applications of the ABI and AHI data.

The developers of SIFT intend that its users and core capabilities will continue to expand, in part based on the initial development principles. The source code is openly available for community development. SIFT also relies heavily on other open-source Python packages and libraries, including SatPy, which will expand in its ability to handle new and additional meteorological satellite instruments and their data formats in the future. SIFT is intended for any interested user in the meteorological community regardless of previous experience with satellite data. Even though the current core users are operational meteorologists and atmospheric scientists primarily in the earth science satellite community, the success of SIFT depends on adoption by the international community and intradisciplinary groups within the atmospheric and oceanic sciences.

Acknowledgments. The authors thank Jessica Braun, Kathleen Strabala, and William Straka III for using and testing SIFT early in development to assess its suitability for deployment. The authors further thank the anonymous peer reviewers during the coronavirus pandemic for casting their thoughtful critiques as minor revisions. The United States National Weather Service (NWS) funded the initial development of SIFT through National Oceanic and Atmospheric Administration (NOAA) grant NA15NES4320001 for the Cooperative Institute for Meteorological Satellite Studies (CIMSS). The views, opinions, and findings contained in this report are those of the authors and should not be construed as an official NOAA or United States government position, policy, or decision. 


\section{APPENDIX A}

\section{Creating the "Night Fog" Brightness Temperature Difference with SIFT}

This appendix contains a brief tutorial for readers interested in creating the "Night Fog" brightness temperature difference and interrogating the corresponding spectral band imagery. To display imagery using SIFT, users must first acquire data then open it. Steps 1 through 3 outline this process for a new user. Steps 4 through 8 demonstrate the useful features of SIFT that are responsible, in part, for Fig. 3 and Fig. 4.

These steps do not include the installation instructions for SIFT. SIFT installers for Windows, Mac, and RedHat/CentOS Linux are available at sift.ssec.wisc.edu/, while software developers can contribute code to the SIFT base through GitHub at github.com/ssec/sift/. Users can download GOES-R ABI data to load with SIFT from NOAA CLASS at www.class.noaa.gov/.

1. Request the data from NOAA CLASS at www.class.noaa.gov/ using the "Search for data" engine. SIFT users should order "GOES-R Series ABI Products (GRABIPRD) (partially restricted L1b and L2+ Data Products)", which is part of the "Environmental Data from Geostationary Satellites". The product type that SIFT reads is "Cloud and Moisture Imagery" (single band). For the subsequent steps, and to follow the example demonstrated in the text of this article, request Band 7 and Band 13 data $(3.9 \mu \mathrm{m}$ and $10.3 \mu \mathrm{m}$, respectively) from either GOES-16 or GOES-17. The GOES-16 "Night Fog" imagery difference in the text used L2 Cloud and Moisture Imagery from 0802 UTC on 1 June 2018.

2. Follow the instructions received from CLASS to download the requested data.

3. After SIFT has started, load the downloaded data from the "Open..." item under the top-level "File" menu in the main SIFT window, click the "+" button to add files to load, and navigate to the correct data storage directory in the file selection window. Choose the desired files and select the "Next" button to proceed. On the next page, choose the desired files (in this case, bands) to load using the checkboxes adjacent to the items. Select "Finish" to load the data.

4. To create a band difference or other arithmetic field, select "Create Algebraic" under the top-level "Layer" menu. A window will open that enables the user to select input fields to create the difference field. Once finalized, SIFT will create a difference field for each of the times in which all of the input fields are loaded. For example, if a user has four different times for which the two bands comprising the difference are available, SIFT will generate four band differences, one for each available time.

5. To change the color enhancement and modify the software-defined minimum and maximum values over which to apply the color enhancement, right click on one of the new difference fields in the "Layers" window, and choose "Change Colormap...".

6. Using the "Change Colormap" window, choose a different colormap for the image using the dropdown menu. The dropdown menu contains those that are included with SIFT. Many are similar or identical to those found in AWIPS. For this "Night Fog" band difference, select the "Fog" enhancement, or create a new variant using the "Gradient Editor", under the top-level "Edit" menu.

7. Before closing the window, modify the minimum and maximum values to best enhance the field on the map display. For the "Night Fog" brightness temperature difference, a typical range is between $-15^{\circ} \mathrm{C}$ and $15^{\circ} \mathrm{C}$. The adjusted range and new colormap will be applied to other loaded layers of the same brightness temperature difference valid at different times.

8. To create the density plot that is shown in the background of Fig. 4, click on the "Region" button in the main SIFT window, and then drop vertices in the map display with left clicks to define the geographic polygon of interest. Close the polygon by choosing a final point near to or on the initial point. This will initially create a bar graph in the "Area Probe Graphs" window. To convert the bar graph to a density plot, select the checkbox next to "Y layer". There are two menus of loaded layers ("X layer" and "Y layer") that enable a pixel-wise comparison for different bands based on the user selection. 


\section{REFERENCES}

Bessho, K., and Coauthors, 2016: An introduction to Himawari-8/9 - Japan's new-generation geostationary meteorological satellites. J. Meteorol. Soc. Japan. Ser. II, 94, 151-183, CrossRef.

Campagnola, L., and Coauthors, 2018: vispy/vispy: Version 0.5.3, CrossRef. (Accessed January 18, 2019).

d'Entremont, R. P., and L. W. Thomason, 1987: Interpreting meteorological satellite images ssing a color-composite technique. Bull. Amer. Meteorol. Soc., 68, 762-768, CrossRef.

Ellrod, G. P., 1995: Advances in the detection and analysis of fog at night using GOES multispectral infrared imagery.

Weather Forecast., 10, 606-619, CrossRef.

Gerth, J. J., 2019: It's not hot air: Using GOES-16 infrared window bands to diagnose adjacent summertime air masses. Meteorol. Appl., 26, 362-368, CrossRef.

Hunter, J. D., 2007: Matplotlib: A 2D graphics environment. Comput. Sci. Eng., 9, 90-95, CrossRef.

Kalluri, S., and Coauthors, 2018: From photons to pixels: Processing data from the Advanced Baseline Imager. Remote Sens., 10, 177, CrossRef.

Klein, A., and Coauthors, 2018: imageio/imageio: V2.4.1, CrossRef.

Lam, S. K., A. Pitrou, and S. Seibert, 2015: Numba: a LLVM-based Python JIT compiler.

Proceedings of the Second Workshop on the LLVM Compiler Infrastructure in HPC - LLVM '15, New York, New York, USA, ACM Press, 1-6, CrossRef.

Lensky, I. M., and D. Rosenfeld, 2008: Clouds-AerosolsPrecipitation Satellite Analysis Tool (CAPSAT). Atmos. Chem. Phys., 8, 6739-6753, CrossRef.

Okuyama, A., M. Takahashi, K. Date, K. Hosaka, H. Murata, T. Tabata, and R. Yoshino, 2018: Validation of Himawari-8/AHI radiometric calibration based on two years of in-orbit data. J. Meteorol. Soc. Japan. Ser. II, 96B, 91-109, CrossRef.

Oliphant, T. E., 2006: A guide to NumPy, Trelgol Publishing, 371 pgs, CrossRef.

Raspaud, M., and Coauthors, 2018a: PyTroll: An open source, community driven Python framework to process Earth Observation satellite data. Bull. Amer. Meteorol. Soc., BAMS-D-17-0277.1, CrossRef. , and Coauthors, 2018b: pytroll/satpy: Version 0.11.0, CrossRef. (Accessed January 18, 2019).

Schmit, T. J., M. M. Gunshor, W. P. Menzel, J. J. Gurka, J. Li, and A. S. Bachmeier, 2005: Introducing the Next-Generation Advanced Baseline Imager on GOES-R. Bull. Amer. Meteorol. Soc., 86, 1079-1096, CrossRef. , P. Griffith, M. M. Gunshor, J. M. Daniels, S. J. Goodman, and W. J. Lebair, 2017: A Closer Look at the
ABI on the GOES-R Series. Bull. Amer. Meteorol. Soc., 98, 681-698, CrossRef.

, S. S. Lindstrom, J. J. Gerth, and M. M. Gunshor, 2018: Applications of the 16 spectral bands on the Advanced Baseline Imager (ABI). J. Oper. Meteorol., 6, 33-46, $\underline{\text { CrossRef. }}$

Space Science and Engineering Center, University of Wisconsin-Madison, 2019: Satellite Information Familiarization Tool (SIFT), CrossRef.

Whitaker, J., pyproj - pyrex generated python interface to PROJ.4 library, CrossRef. 